\title{
The development of a rescheduling system for train traffic operation
}

\author{
H. Nakajima, A. Ishikawa, M. Nakamura \& F. Henda \\ East Japan Railway Company, Japan
}

\begin{abstract}
In this study, by analyzing what the actual rescheduling dispatcher does and constructing an algorithm, we have developed a system to propose rescheduling, and verified the performance for practicality. In addition, to get closer to practical use, we rebuilt the algorithm and system, setting that the timing of the proposal is the time of resuming the entire operation, and verified it by using the data of actual dispatcher training.

Keywords: rescheduling, dispatcher, algorithm, ATOS, propose rescheduling, actual dispatcher training, resuming entire operation.
\end{abstract}

\section{Introduction}

In the Tokyo Metropolitan area of Japan, the railway radiates from the circular Yamanote Line to the suburbs. There are also many lines of subway inside the Yamanote Line. These lines of this area form a big complex network and have the mass and high density transport. In order to achieve this high-density transport, we scrutinize in detail train stoppage times and the train travelling times according to equipment condition and past experience, and carefully set up a schedule. However, if trains cannot be operated as planned (we call it "diagram is confusing") due to problems such as equipment failure or injury or accident, the dispatcher changes the destination of the train, aborts the operation to secure the amount of transportation, recovers the delay and reduces confusion for customers.

Changing the plan is called "rescheduling". This is performed by the dispatcher who has been monitoring the situation. In a state of on-time operation, the work of dispatchers has come to be almost unnecessary due to the evolution of IT in recent years. However, if the system has not yet advanced into a state of 
diagram confusion, then dispatchers have to change the schedule based on their hunches and experiences.

So far, the function of partially supporting rescheduling that includes changing the order or changing the siding station has been developed [1]. However, because it does not take the overall train schedules into account and does not provide an organized operation, it is hard to say that it provides enough assistance. To improve the contents for further support, we are developing the system to make the proposal of entire rescheduling until the delay is eliminated, by adding functionality judgment of the suspended train, changing terminus, or setting on a special train with consideration of availability to our customers.

When dispatchers reschedule, they have to confirm that those changes of diagram do not cause any trouble to the cars and crews management. These confirmations are executed through managers for cars and crews, by cooperating with train depots and crew offices. Therefore, by connecting the system that supports the management of cars and crews to the rescheduling system, dispatchers can see the constrained conditions of periodic inspections and the existence of crews (drivers or conductors) who are supposed to get on, and the system can also manage a chain of operations comprehensively at the same time (Figure 1).

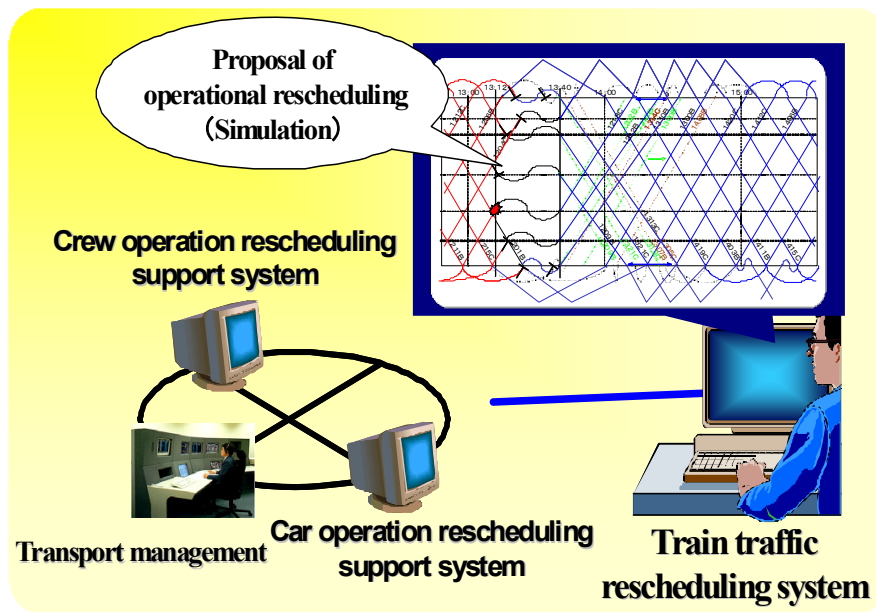

Figure 1: Basic concept of rescheduling system.

\section{Development of algorithm for rescheduling}

In this study, the system was aimed for the lines of the ATOS (Autonomous decentralized Transport Operation control System) area. We developed the system at The Chuo-Sobu Local line as a model line, where we have already developed the train cars and crews management support systems so that it will be possible to work with them [2]. 
We have to cancel the operation of trains to make delays disappear. But if we cancel many operations, it would be difficult to secure the transport capacity of passengers who have been waiting on platforms until operations resume. It then becomes necessary to run extra trains. Therefore, we think that the algorithm should forecast the timing when passengers who are waiting on platforms will be able to get on the train based on the number of daily ordinary passengers in each section and on the number of trains that can be run at the time zone concerned on the forecasted diagram. This algorithm is expected to work out appropriate rescheduling according to transportation demand.

\subsection{Evaluating logic for diagram}

We took the following logic to evaluate the diagram objectively by quantification, using data of all traffic research for local trains.

- Divide a diagram into areas by a unit of minimum headway to evaluate transport ability in each hour in detail.

- Divide a diagram into sections of main stations to focus differences of passengers between each section.

- Treat each area that is divided as mentioned above as a unit to analyze and evaluate.

- Calculate the over-and-shorts number of trains based on the number of remaining passengers to evaluate the number of trains in each area (Figure 2).

- Calculate the differences of headway between the original diagram and the forecasted diagram by each area to evaluate headway between trains in each area.

- Calculate the maximum minutes of delay and the total delay that is the accumulation of delay of each train to evaluate delay of trains in each area.

- Calculate the number of trains that are indicated as delayed for priority trains in each area.

- We evaluated the diagram by using the values mentioned above, such as the over-and-shorts number of trains, increase of headway, delays, and so on.

\subsection{Algorithm for rescheduling}

\subsubsection{Basic algorithm}

We set the algorithm to determine the best operation (for example, turning back or cancellation) to achieve a target in all data of improving the target calculated by the forecasted diagram, diagram evaluating, and so on. This algorithm also applies those operations, evaluates, proposes the diagram if it has been improved, and does these calculations, until the delays disappear within the forecast. We consider this algorithm as basic (Figure 3). 


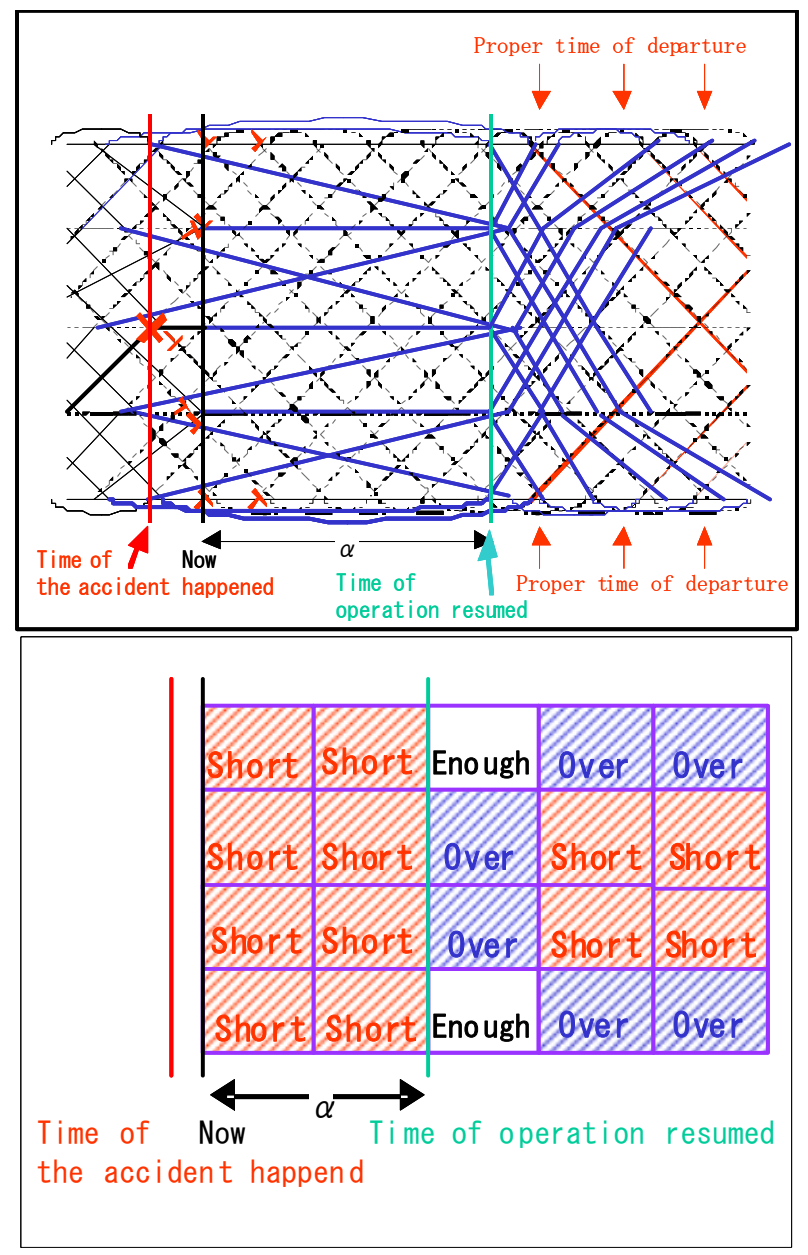

Figure 2: Confusion diagram (top) and evaluation of those areas (bottom).

\subsubsection{Practical algorithm}

In addition to the basic algorithm, we developed a practical algorithm by knowhow based on dispatchers. Those are dispatcher's operations, such as changing the departing order, turning back before the terminal station, going into train burg, getting out from train burg, extending the last stop, setting temporary trains and changing connections between trains.

As one example of dispatcher's operations, we show the example of changing the departing order of the trains that return to the terminal, if there is a difference of delays between both directions (Figure 4). 


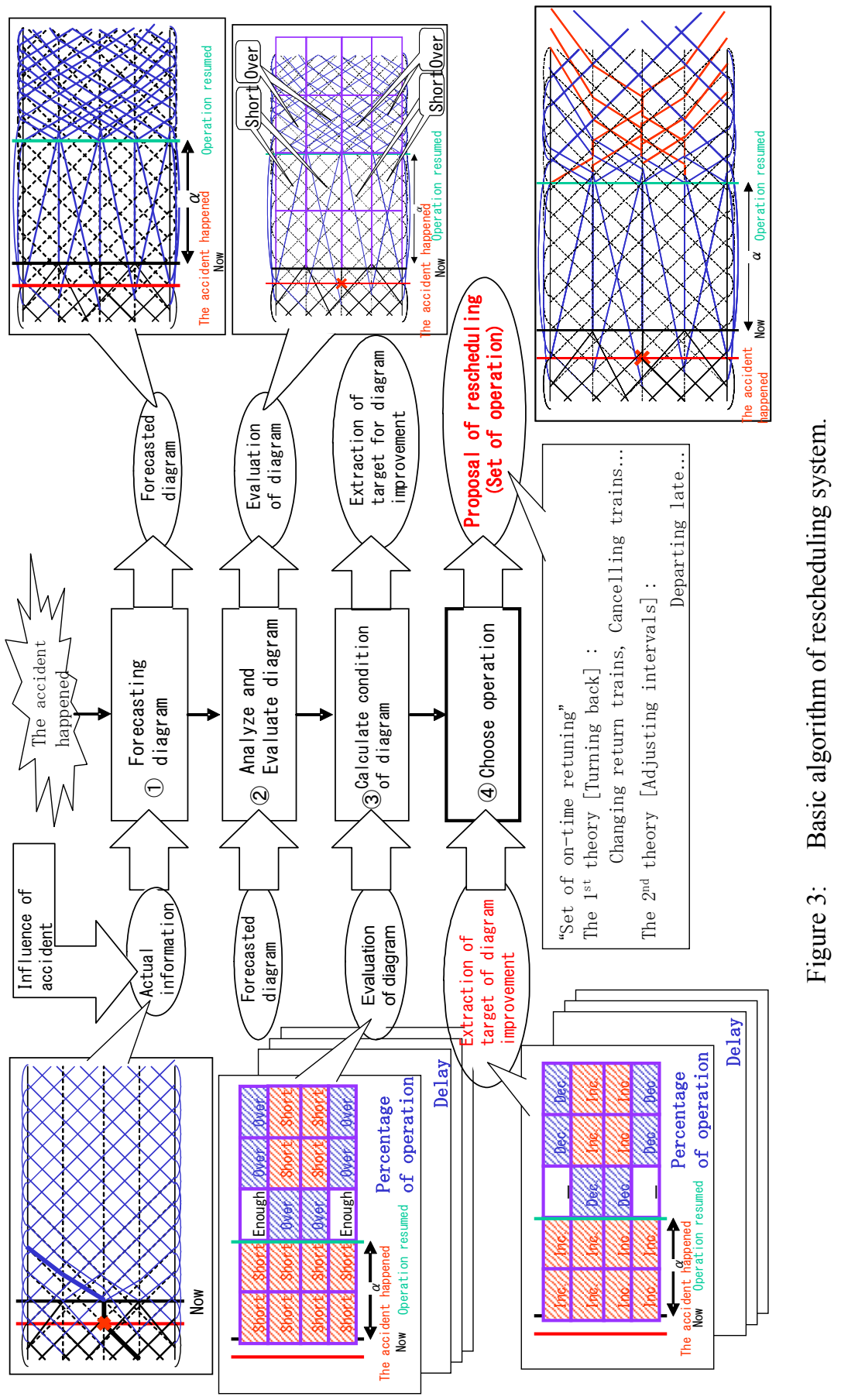

WIT Transactions on The Built Environment, Vol 135, (C) 2014 WIT Press www.witpress.com, ISSN 1743-3509 (on-line) 


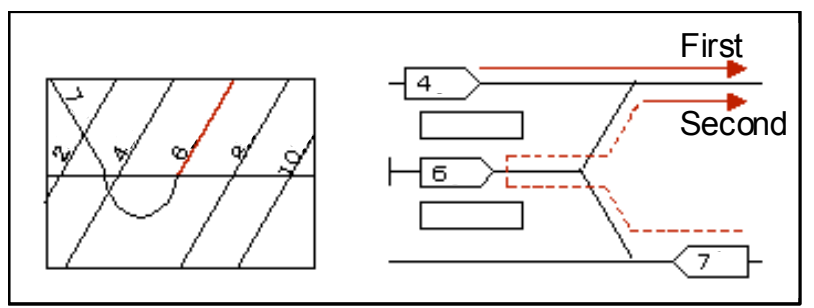

Figure 4: Changing departing order at the returning station.

If the up train No. 4 is delayed and the down train No. 7 is not delayed, No. 6 train that has front connection to No. 7 cannot depart until No. 4 train departs. Therefore, they operate by changing the departing order so that No. 4 train departs later, and No. 6 train departs earlier. In another case, if the up trains are not delayed and the down train No. 7 is delayed, No. 8 train cannot depart until No. 7 train arrives and No. 6 train departs. Therefore, they operate by changing the departing order so that No. 6 train departs later, and No. 8 train departs earlier.

Conditions for systemization:

- The turning train is delayed at the starting station due the delay ahead.

- The train that runs behind is delayed at the turning station due to the delay of the turning train.

The equation below shows these trains:

(Forecasted departure time of train departing later)

$=($ Forecasted departure time of train that is delayed at the starting station $)$

+ (Minimum headway at that line)

\subsubsection{The colour displaying function}

We also developed a function that shows the degree of congestion of the trains and numbers of passengers who would not be able to get into the carriages. This is shown by colours on a proposed diagram that simulates the rescheduling system. By pushing the button displayed as "mesh display width" on the display, we can set a time width to divide the diagram into areas. As a minimum unit of that time width, the system calculates the mean values of waiting passengers' level of each unit (Table 1).

The calculated mean values are displayed at each areas divided into sections of stations and times (Figure 5) by using colours shown in Table 2. 
Table 1: Levels of waiting passengers.

\begin{tabular}{|l|l|}
\hline Level & Number of waiting passengers \\
\hline Level 0 & $0-$ Normal number \\
\hline Level 1 & Normal number $-2,999$ \\
\hline Level 2 & $3,000-3,749$ \\
\hline Level 3 & $3,750-4,499$ \\
\hline Level 4 & $4,500-5,249$ \\
\hline Level 5 & $5,250-5,999$ \\
\hline Level 6 & $6,000-6,749$ \\
\hline Level 7 & $6,750-7,499$ \\
\hline Level 8 & $7,500-8,249$ \\
\hline Level 9 & $8,250-8,999$ \\
\hline Level 10 & 9,000 or more \\
\hline
\end{tabular}

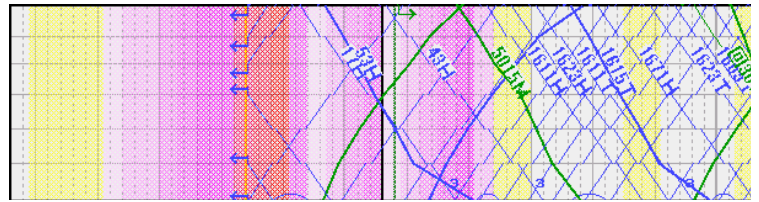

Figure 5: The colour display (the mesh width is 90 seconds).

Table 2: Colours for each level.

\begin{tabular}{|l|l|}
\hline Level of waiting passengers & Colour \\
\hline Level 0 & \\
\hline Level 1 & \\
\hline Level 2 & \\
\hline Level 3 & \\
\hline Level 4 & \\
\hline Level 5 & \\
\hline Level 6 & \\
\hline Level 7 & \\
\hline Level 8 & \\
\hline Level 9 & \\
\hline Level 10 & \\
\hline
\end{tabular}

\section{Development of off-line test equipment}

\subsection{Construction of test equipment}

We included the algorithm of the rescheduling system that we have developed into the simulator of GD (Graphic Display) which is input equipment for ATOS. We constructed test equipment in order that we can test various cases by using it, which is the mock of an actual diagram (arrival and departure times) from an off-line EDP (Electric Data Processer) simulator. (Figure 6) The test equipment was installed in the training room for dispatchers. 


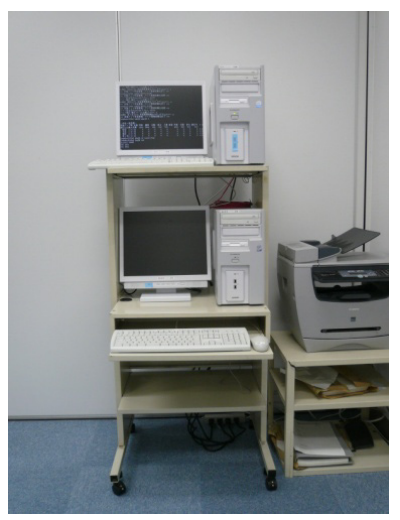

Figure 6: Rescheduling system.

\subsection{The results of the off-line test}

The time to recovery, total delay minutes, headway of trains, the number of delayed trains and the number of cancelled trains have been rated by dispatchers as correct. We compared those numerical values with the dispatchers' values more concretely in this off-line test. We also compared the operated percentage (the ratio of operated trains compared with planned schedule) and on-time operated percentage (the ratio of delay trains compared with planned schedule).

The results are shown below.

\section{1) The plan of rescheduling}

Dispatchers operated as "move trains if possible" or "stop trains at once" according to the situation of an accident, and tried to keep transport capacity even though there was an obstacle caused by the accident. On the other hand, the system mostly chose "stop at once", which makes the maximum transport capacity after operations are resumed (Figure 7).

2) The total delay minutes

The total delay minutes of dispatchers showed a tendency to be smaller because they moved trains as much as possible. But those of the system are bigger than those of the dispatchers because the system chose "stop at once", which made all the trains late while operations were stopped.

3) The headway

Dispatchers stopped the trains at the last stations to which they can advance, so after operations resumed, headway was quite variable and the delays at the returning station sometimes increased. On the other hand, the system showed the same headway after operations resumed.

4) The number of delayed trains, the number of cancelled trains and time to return to planned schedule

There was no great difference between the dispatchers' rescheduling and the system's. The reason for this is that operations were stopped for about 1 hour due to an accident resulting in injury or death, and they easily guessed the time that operations would be resumed. It was comparatively light rescheduling. 
$<$ An example of "Move trains if possible". $>$

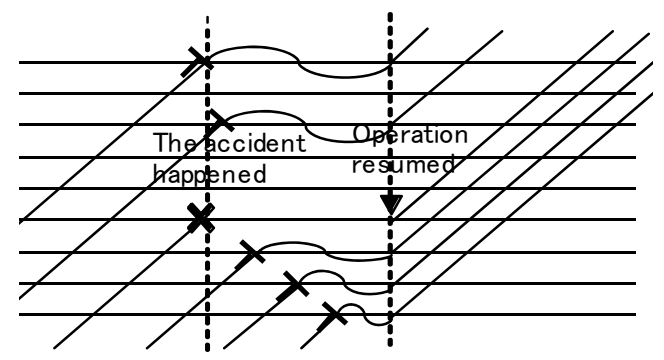

$<$ An example of "Stop trains at once". >

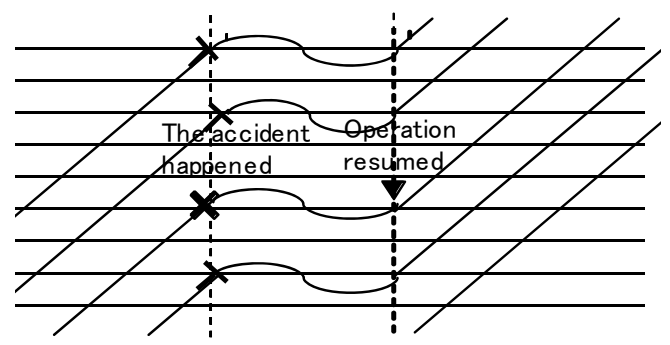

Figure 7: "Move trains if possible" and "Stop trains at once".

5) Percentage of operated trains, and percentage of on-time trains

Dispatchers flexibly decided to cancel operations or to change returning station according to the place where the accident happened, so there was a great difference in percentage of operated trains and percentage of on-time trains between the dispatchers' rescheduling and the system's depending on sections. However, there was not a great difference between the two percentage by the system because influence was equalized over the whole line.

\section{The improvement of rescheduling the algorithm and connecting to the ATOS training system}

In the test described in the last chapter, we built the system program as a standalone rescheduling system, and we tested validity of rescheduling proposals of the system by comparing the results with the operations that the dispatchers did during training. The first action when the accident happened was that dispatchers moved trains as much as possible, but the rescheduling system chose "stop at once". That is, there was a big difference between the operation that the rescheduling system proposed and the operation that the dispatchers did as their first action.

In fact, time when operations resumed was uncertain at the step of first action, and the actual running conditions of trains were different from the simulation of 
the rescheduling system. So, a precondition of comparison was greatly different. Furthermore, compared with dispatchers' rescheduling, total delay minutes of the system proposal had a tendency to be large. Therefore, the rescheduling that the system proposed did not have the advantage. However, though first actions had many differences, subsequent rescheduling proposals to recover delay were mostly appropriate compared with dispatchers.

\subsection{The improvement of rescheduling the algorithm}

We improved the rescheduling algorithm to propose the reschedule, which was closer to actual management, and to get the practical proposal. Generally, many decisions need to be taken by humans as the first action for a train accident. Consequently, the whole rescheduling from first action for an accident by the system does not match the present train operation. It is also difficult to guess the time when trains would be resumed, because the timing is changeable depending on conditions. Therefore, we decided that we start the rescheduling system with precedence of human decisions, and we aimed for supporting the train operation.

\subsection{Connecting to the ATOS training system}

As a measure to get actual arrival and departure times for rescheduling according to the dispatchers' first action, we utilized ATOS training equipment.

Dispatchers train regularly for accidents by using the system for the purpose of progressing ability (about four times a month). The training system has the same equipment as the actual input equipment of ATOS, and is capable of simulating situations of train accidents by imitating the running of trains.

It was hard to gain actual arrival and departure times from the actual ATOS system to the rescheduling system, so in this step we set the rescheduling system so that it can gain time data of the dispatcher's first action from the ATOS training system.

\subsection{System configuration}

Figure 8 shows the system configuration of rescheduling system.

\section{Verification and consideration of rescheduling diagrams}

We worked the rescheduling system many times when ATOS dispatchers trained for the Chuo-Sobu Local Line, and got results of proposed rescheduling. The rescheduling system was confirmed to show the proposals in about 3-5 minutes. (Occasionally, it did not show the proposals depending on the first action of dispatchers.)

The rescheduling system almost worked out proper proposals, but partly the system could not solve pending connections between trains that dispatchers left. Trains that have pending connections have possibly various ways of operation, such as turning back and coming into or out from train shed, which will change 
later depending on the delay situation. Dispatchers finally decide all connections of trains. However, they do not decide all of them at the initial phase of rescheduling, and the decision is flexible depending on the delay situation. There are normally some pending connections at the moment when operations resumed (Figure 9).

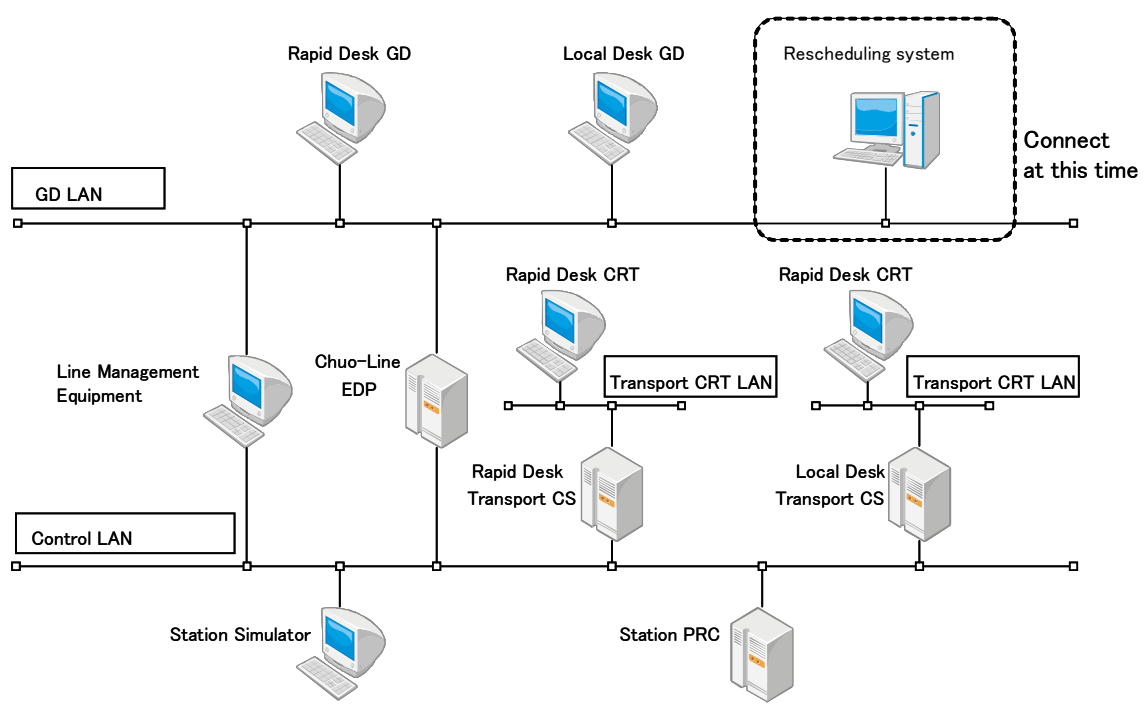

Figure 8: System configuration.

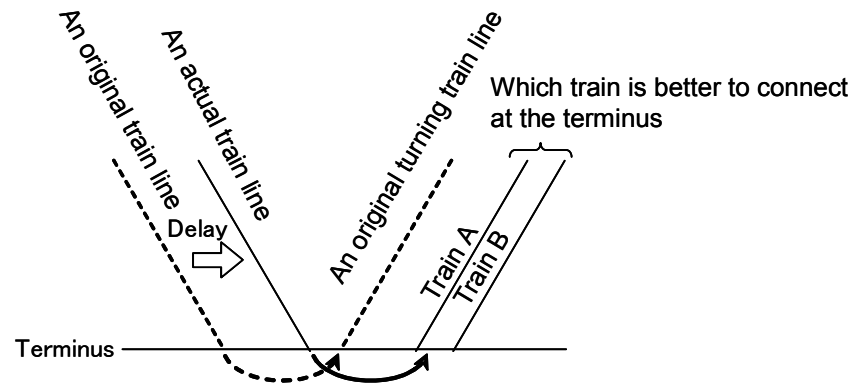

If the train will have no delay or the delay will be decreasing. : Connect to Train A If the delay will be increasing. : Connect to Train B.

Figure 9: The example of the train that makes a pending connection. 
At the beginning, the rescheduling system was developed to use an unchanged diagram. Rescheduling by dispatchers involves temporal irregularity in the diagram, so the system could not solve this automatically.

We think that in the future the system should solve such intentional pending connections by dispatchers.

\section{Conclusion}

At first, the rescheduling system had been developed as the system that proposes a series of rescheduling from accident occurrence to resuming operations and recovering delays at the same time. We improved the system to work in response to the first action of dispatchers with a view to closer use of actual operations. We also joined the rescheduling system to the ATOS training system to gain information of input by dispatchers. This made the rescheduling system able to work with an initial condition generated by the dispatcher's first action during their ATOS training. We will continually compare rescheduling of the system with that of dispatchers to verify the algorithm of the rescheduling system. Based on the verification, we will research problems for practical use in the future.

\section{References}

[1] Railway Technical Research Institute Planning Systems Laboratory, Scheduling algorithms in Railways.

[2] K. Inoue, Y. Arisawa, M. Souma \& F. Henda, Development of train traffic rescheduling system, JR EAST Technical Review No. 20, pp. 59-61, 2007. 